\title{
Measuring Model of Cylindrical Workpiece
}

\author{
Kuijing Zheng \\ Key Laboratory of Advanced Forging \& Stamping \\ Technology and Science (Yanshan Universtiy), \\ Hebei Provincial Key Laboratory of Parallel Robot and \\ Mechatronic System, Yanshan University \\ Qinhuangdao, China \\ kjzheng@ysu.edu.cn
}

\begin{abstract}
Objective: The three-dimensional reconstruction method based on vision uses the digital camera as the image sensor. Establishing the model of a dynamic measured object is the premise of the vision precise measuring system. Methods: By using the pinhole camera model and the mathematical modeling method, the three-dimensional reconstruction of the measured part can be completed quickly based on the known measured objects. The corresponding relation can be realized accurately and automatically on condition that the object's shape characteristic is known clearly. The algorithm of the threedimensional measuring model of cylindrical workpiece can be established conveniently. Results: The algorithms are verified by simulation with Solidworks and Matlab. The correctness of the algorithm is validated by actual experiment and the absolute error is less than $0.015 \mathrm{~mm}$ or the relative error is less than $0.1 \%$. Conclusion: The measuring results are accurate enough to satisfy the practical requirement of fast and precise measuring in industrial field.
\end{abstract}

Keywords- cylindrical workpiece;vision measuring; camera model; mathematical modeling; three-dimensional measuring

\section{INTRODUCTION}

The vision measuring system uses camera to shoot the measured objects and the obtained picture contains the information about the objects in 2-dimensional image plane. We need to restore the information to the real objects in 3-dimensional space. This procedure is 3dimensional reconstruction[1,2]. The 3-dimensional reconstruction method based on vision uses the digital camera as the image sensor. It is divided into monocular, binocular and multiple vision system. The monocular vision includes shading value method[3,4], photometric stereo vision method[5], texture method[6], contour method[7],focusing method[8], motion method [9,10], etc. This vision measuring method doesn't subject to the restriction of object's shape and the speed of reconstruction, so it can be realized automatically or semi-automatically.

Based on the known measured objects, the pinhole image model can be established and the mathematical modeling methods can be used to complete the 3dimensional reconstruction. The cylindrical workpiece will be used as an example to introduce the camera image model, the steps of mathematical modeling and verification by simulation and actual measuring test.

\author{
Fei Xu \\ Key Laboratory of Advanced Forging \& Stamping \\ Technology and Science (Yanshan Universtiy), \\ Hebei Provincial Key Laboratory of Parallel Robot and \\ Mechatronic System, Yanshan University \\ Qinhuangdao, China \\ 776967209@qq.com
}

\section{THE CAMERA IMAGING MODEL}

A. Coordinate system

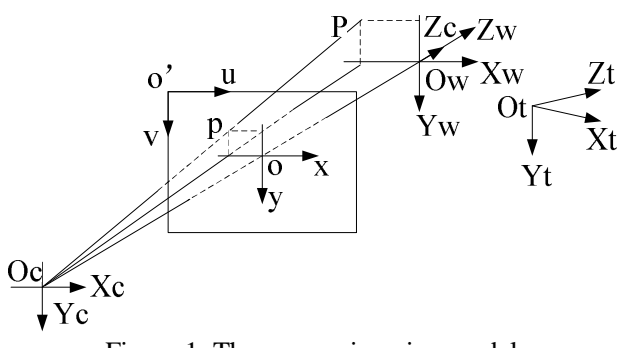

Figure 1. The camera imaging model

As shown in fig. 1, there are five coordinate systems in pinhole imaging model.The imaging coordinate system is $\{I\}, \mathrm{o}-\mathrm{xy}$. It is within the imaging plane corresponding to CCD plane of the camera. The unit is ' $m$ '. The main point ' $\mathrm{o}$ ' is the intersection of the camera's main light and the plane. The camera coordinate system is $\{C\}$, Oc$\mathrm{X}_{\mathrm{c}} \mathrm{Y}_{\mathrm{c}} \mathrm{Z}_{\mathrm{c}} \cdot \mathrm{O}_{\mathrm{c}}$ is the optic center, $\mathrm{O}_{\mathrm{c}} \mathrm{Z}_{\mathrm{c}}$ is the direction of the main beam and the length of $\mathrm{O}_{\mathrm{c}} \mathrm{O}$ is the principal distance $f$. The world coordinate system is $\{W\}, \mathrm{O}_{\mathrm{w}}-X_{\mathrm{w}} Y_{w} Z_{\mathrm{w}}$. The image coordinate system is $\left\{I^{\prime}\right\}, \mathrm{o}^{\prime}-\mathrm{uv}$. The unit is pixel. The origin $o^{\prime}$ is the upper left point of the image. The object coordinate system is $\{T\}, \mathrm{O}_{\mathrm{t}}-\mathrm{X}_{\mathrm{t}} \mathrm{Y}_{\mathrm{t}} \mathrm{Z}_{\mathrm{t}}$. The angle between $\mathrm{O}_{\mathrm{t}} \mathrm{Z}_{\mathrm{t}}$ and $\mathrm{O}_{\mathrm{c}} \mathrm{Z}_{\mathrm{c}}$ is the angle between the light projected onto the object and the main optical axis.

\section{B. Conversion relationship}

Space point in $\{\mathrm{C}\}:{ }^{C} \boldsymbol{P}=[\mathrm{Xc}$ yc zc 1$] \mathrm{T}$; Space point in $\{\mathrm{W}\}:{ }^{W} \boldsymbol{P}=[\mathrm{XW}$ yw $\mathrm{zW} 1] \mathrm{T}$; Image point in $\{\mathrm{I}\}: \boldsymbol{m}=\left[\begin{array}{lll}\mathrm{X} & \mathrm{y} & 1\end{array}\right] \mathrm{T}$; Image point in $\left\{\mathrm{I}^{\prime}\right\}: \boldsymbol{m}^{\prime}=\left[\begin{array}{lll}\mathrm{u} & \mathrm{V} & 1\end{array}\right] \mathrm{T}$.

It can be inferred that ${ }^{C} \boldsymbol{P}$ and its image point $\mathrm{m}$ satisfy the following relationship:

$z_{c} \boldsymbol{m}=\boldsymbol{M}^{C} \boldsymbol{P}$

where $\boldsymbol{M}$ is the camera matrix and $\mathrm{f}$ is the focal length. follows

The transformation between $\left\{\mathrm{I}^{\prime}\right\}$ and $\{\mathrm{I}\}$ is as

$$
m^{\prime}=S \mathbf{m}
$$

where $S$ is the image transformation matrix, u0 and $\mathrm{v} 0$ are the coordinate of camera principal points in the $\mathrm{U}$ axis and $\mathrm{v}$-axis direction of the $\left\{\mathrm{I}^{\prime}\right\}$ (pixel), 
$\mathrm{sx}=\mathrm{Lx} / \mathrm{px}(\mathrm{m} / \mathrm{pixel})$ and $\mathrm{sy}=\mathrm{Ly} / \mathrm{py}(\mathrm{m} / \mathrm{pixel})$ are the scaling factors of the camera in the u-axis and v-axis, Lx and Ly are the width and height of CCD, px and py are the pixel values in the photo width and height direction.

The relationship between $\{\mathrm{W}\}$ and $\{\mathrm{C}\}$ is as follows

$$
{ }^{C} \boldsymbol{P}=\left[\begin{array}{ll}
\boldsymbol{R} & \boldsymbol{t} \\
\boldsymbol{0} & 1
\end{array}\right]{ }^{W} \boldsymbol{P}
$$

Where $\boldsymbol{R}$ is the rotation matrix, $\alpha, \beta, \gamma$ are rotation angles, $t=[\mathrm{tx}$ ty tz] is the translation vector, tx ty tz are the translation value.

Then

$$
z_{c} \boldsymbol{m}^{\prime}=\boldsymbol{K}\left[\begin{array}{ll}
\boldsymbol{I} & 0
\end{array}\right]\left[\begin{array}{ll}
\boldsymbol{R} & \boldsymbol{t} \\
\boldsymbol{0} & 1
\end{array}\right]{ }^{\mathrm{W}} \boldsymbol{P}
$$

where $\left[\begin{array}{ll}\boldsymbol{R} & \boldsymbol{t}\end{array}\right]$ is the camera's external parameters matrix, $\boldsymbol{K}$ is the camera's internal parameters matrix, ax is $\mathrm{f} / \mathrm{sx}$, ay is $\mathrm{f} / \mathrm{sy}$.

\section{THE STEPS OF MATHEMATICAL MODELING}

The flow chart of the mathematical modeling is specified as in Fig. 2 .

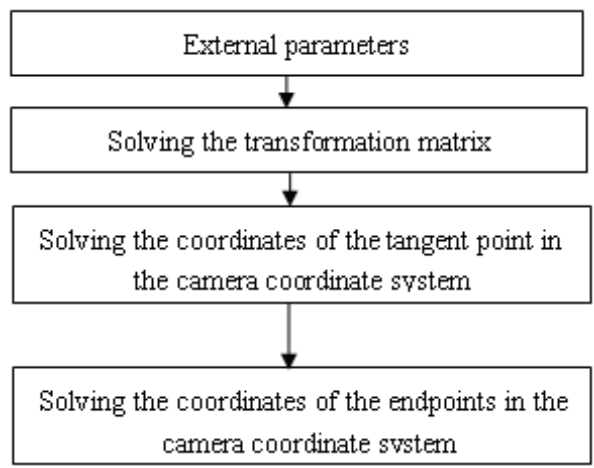

Figure2. The flowchart of mathematical modeling

(1)Determination of external parameters: The camera's external parameters matrix contains three rotation variables such as $\alpha, \beta, \gamma$ and three translation variables such as $t_{x}, t_{y}, t_{z}$. These variables will have effect on the imaging.

(2) Solving the transformation matrix between $\{T\}$ and $\{C\}$.

(3) Solving the coordinate of the tangent point of the measured object in $\{C\}$. First we solve the representation of the tangent point in $\{T\}$ and then convert it to the representation in $\{C\}$. We analyze the surface perpendicular to the measured object's surface and define the intermediate variables and simplify the calculation and the expression.

(4) Solving the coordinates of the endpoint of the measured object in $\{C\}$. First we solve the representation of the intermediate point in $\{C\}$. Then we solve the expression of the upper and lower endpoints.

\section{MATHEMATICAL MODELING OF CYLINDRICAL WORKPIECE}

We establish the mathematical model to obtain the length of a cylinder assumed that its radius $R$ is known in monocular vision measuring system. The external parameters include the rotation value $\beta$ of the cylinder around $Z_{c}$-axis and the translation value $s_{y}$ along $Y_{c}$-axis.

\section{A. Solving the transformation matrix}

The solution for the transformation matrix between $\{T\}$ and $\{C\}$ is shown in Fig.3.

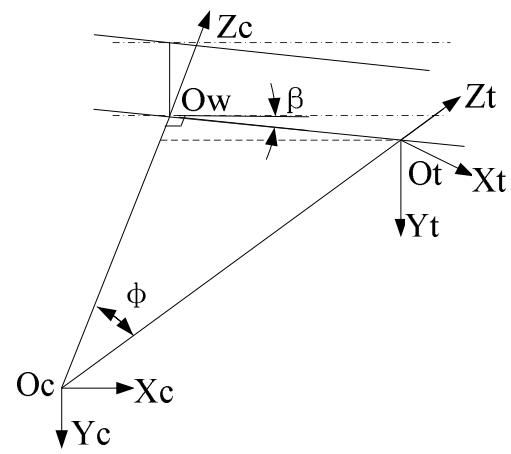

Figure 3. The transformation matrix between $\{T\}$ and $\{C\}$

In Fig. 3, $O_{w} O_{t}$ is equal to $L_{x}$ and $O_{c} O_{w}$ is equal to $s$. The transformation from $\{T\}$ to $\{C\}$ includes three steps: first translates $L_{x} \cdot \cos \beta$ along $X_{c}$-axis, second translates $s$ $L_{x} \cdot \sin \beta$ along $Z_{c}$-axis, third rotates $\varphi$ around $Y_{c}$-axis. Then the transformation matrix is as follows

$$
{ }_{T}^{C} T=\left[\begin{array}{cccc}
\cos \varphi & 0 & \sin \varphi & L_{x} \cdot \cos \beta \\
0 & 1 & 0 & 0 \\
-\sin \varphi & 0 & \cos \varphi & s-L_{x} \cdot \sin \beta \\
0 & 0 & 0 & 1
\end{array}\right]
$$

where $\sin \varphi=L_{x} \cdot \cos \beta / \sqrt{\left(L_{x} \cdot \cos \beta\right)^{2}+\left(s-L_{x} \cdot \sin \beta\right)^{2}}$,

$$
\cos \varphi=\left(s-L_{x} \cdot \sin \beta\right) / \sqrt{\left(L_{x} \cdot \cos \beta\right)^{2}+\left(s-L_{x} \cdot \sin \beta\right)^{2}}
$$

\section{B. Solving the tangent point in $\{C\}$}

The solution to solve the upper tangent point $P_{T}$ of the cylinder in $\{T\}$ is shown in Fig.4. The lower tangent point is similar. For $P_{T}$ in $\{T\},{ }^{T} x_{T}=0,{ }^{T} y_{T}=-P_{t} Q,{ }^{T} z_{T}=-Q O_{t}$. $P_{t} Q$ is equal to $P M$ and $Q O_{t}$ is equal to $M J$.

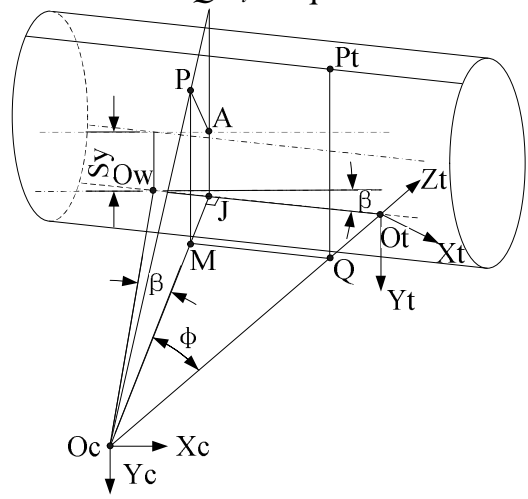

Figure 4.The coordinate of $P_{T}$ the tangent point of the cylinder in $\{T\}$

As shown in Fig.5, the tangent plane perpendicular to the cylindrical surface is selected to analyze. $Q 、 M 、 N$ are pedals of the middle tangent point, upper tangent point and lower tangent point on the plane $O_{\mathrm{c}} O_{\mathrm{w}} O_{\mathrm{t}}$. The line $\mathrm{BGD}$ is the image of the cross section on the image plane.

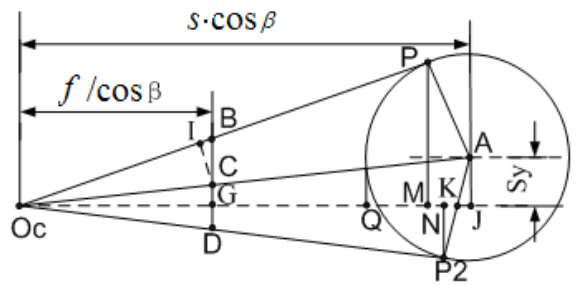

Figure 5. The tangent plane perpendicular to the cylindrical 
Four intermediate variables are defined as follows

$$
\begin{aligned}
& q_{1}=\left(R \sqrt{(s \cdot \cos \beta)^{2}+s_{y}^{2}-R^{2}}-s \cdot s_{y} \cdot \cos \beta\right) /\left((s \cdot \cos \beta)^{2}-R^{2}\right) \\
& q_{2}=\sqrt{(s \cdot \cos \beta)^{2}+s_{y}^{2}-R^{2}} \\
& q_{3}=\sqrt{(s \cdot \cos \beta)^{2}+\left(L_{x}-s \cdot \sin \beta\right)^{2}} \\
& q_{4}=\left(R \sqrt{(s \cdot \cos \beta)^{2}+s_{y}^{2}-R^{2}}+s \cdot s_{y} \cdot \cos \beta\right) /\left((s \cdot \cos \beta)^{2}-R^{2}\right)
\end{aligned}
$$

The upper tangent point in $\{T\}$ is obtained

$$
{ }^{T} \boldsymbol{P}_{T}=\left[\begin{array}{c}
0 \\
-q_{1} \cdot q_{2} / \sqrt{q_{1}^{2}+1} \\
q_{2} \cdot q_{3} /\left(s \cdot \cos \beta \sqrt{q_{1}^{2}+1}\right)-q_{3}
\end{array}\right]
$$

According to formula (5) and formula (6), the upper tangent point of the cylinder in $\{C\}$ is as follows

$$
{ }^{c} \boldsymbol{P}_{T}=\left[\begin{array}{c}
L_{x} \cdot q_{2} /\left(s \cdot \sqrt{q_{1}^{2}+1}\right) \\
-q_{1} \cdot q_{2} / \sqrt{q_{1}^{2}+1} \\
q_{2}\left(s-L_{x} \cdot \sin \beta\right) /\left(s \cdot \cos \beta \sqrt{q_{1}^{2}+1}\right)
\end{array}\right]
$$

Similarly, the lower tangent point of the cylinder in $\{C\}$ is as follows

$$
{ }^{C} \boldsymbol{P}_{B}=\left[\begin{array}{c}
L_{x} \cdot q_{2} /\left(s \cdot \sqrt{q_{4}^{2}+1}\right) \\
q_{4} \cdot q_{2} / \sqrt{q_{4}^{2}+1} \\
q_{2}\left(s-L_{x} \cdot \sin \beta\right) /\left(s \cdot \cos \beta \sqrt{q_{4}^{2}+1}\right)
\end{array}\right]
$$

\section{Solving the endpoint in $\{\mathrm{C}\}$}

The equations are an exception to the prescribed specifications of this template. You will need to determine whether or not your equation should be typed using either the Times New Roman or the Symbol font (please no other font). To create multileveled equations, it may be necessary to treat the equation as a graphic and insert it into the text after your paper is styled.

The solution to solve the endpoints of the cylinder in $\{C\}$ is shown in Fig.6. $M P_{M}$ is equal to $L_{R}, M_{1} M_{2}$ is also equal to $L_{R}$ and $M_{3} M_{4}$ is equal to $s_{y}$.

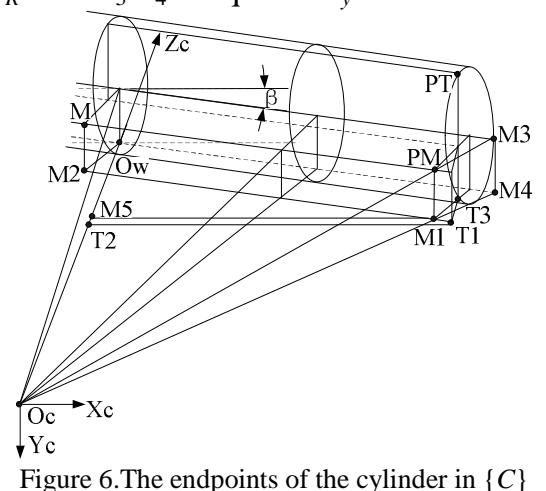

The middle endpoint in $\{C\}$ is obtained

$$
{ }^{C} P_{M E}=\left[\begin{array}{c}
L_{R} \cdot \cos \beta-R \cdot s \cdot \sin \beta \cdot \cos \beta / \sqrt{(s \cdot \cos \beta)^{2}+s_{y}^{2}} \\
s_{y}\left(1-R / \sqrt{(s \cdot \cos \beta)^{2}+s_{y}^{2}}\right) \\
s-L_{R} \cdot \sin \beta-R \cdot s \cdot \cos ^{2} \beta / \sqrt{(s \cdot \cos \beta)^{2}+s_{y}^{2}}
\end{array}\right]
$$

The upper endpoint and the lower endpoint in $\{C\}$ are as follows

$$
{ }^{C} P_{T E}=\left[\begin{array}{c}
L_{R} \cdot \cos \beta+\sin \beta \cdot q_{2} / \sqrt{q_{1}^{2}+1}-s \cdot \sin \beta \cdot \cos \beta \\
-q_{1} \cdot q_{2} / \sqrt{q_{1}^{2}+1} \\
q_{2}\left(s-L_{x} \cdot \sin \beta\right) /\left(s \cdot \cos \beta \sqrt{q_{1}^{2}+1}\right)
\end{array}\right]
$$

$$
{ }^{C} P_{B E}=\left[\begin{array}{c}
L_{R} \cdot \cos \beta+\sin \beta \cdot q_{2} / \sqrt{q_{4}^{2}+1}-s \cdot \sin \beta \cdot \cos \beta \\
q_{4} \cdot q_{2} / \sqrt{q_{4}^{2}+1} \\
q_{2}\left(s-L_{x} \cdot \sin \beta\right) /\left(s \cdot \cos \beta \sqrt{q_{4}^{2}+1}\right)
\end{array}\right]
$$

\section{The length of cylinder}

The length of cylinder is obtained by

$$
L=L_{R^{-}} L_{F}
$$

where $L_{R}$ is the length of the right part of the cylinder and $L_{F}$ is the inverse number of the length of the left part of the cylinder.

For the middle endpoint

$$
L_{R}=\frac{s \cdot x_{M}}{f \cdot \cos \beta+x_{M} \cdot \sin \beta}+\frac{R \cdot s \cdot \cos \beta}{\sqrt{(s \cdot \cos \beta)^{2}+s_{y}^{2}}} \cdot \frac{f \cdot \sin \beta-x_{M} \cdot \cos \beta}{f \cdot \cos \beta+x_{M} \cdot \sin \beta}
$$

For the upper endpoint

$$
L_{R}=\frac{\left(\frac{s \cdot x_{T}}{f \cdot \cos \beta+x_{T} \cdot \sin \beta}-s \cdot \sin \beta\right) \sqrt{(s \cdot \sin \beta)^{2}+s_{y}^{2}-R^{2}}}{\cos \beta \sqrt{\frac{\left(R \sqrt{(s \cdot \sin \beta)^{2}+s_{y}^{2}-R^{2}}-s \cdot s_{y} \cdot \cos \beta\right)^{2}}{\left((s \cdot \sin \beta)^{2}-R^{2}\right)^{2}}}+1}+s \cdot \sin \beta
$$

For the lower endpoint

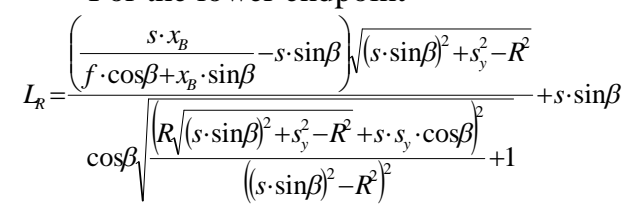

Both of $L_{F}$ and $L_{R}$ are the same expression, just the known conditions are the coordinates of the three left side endpoints in the imaging coordinate.

\section{VERIFICATION}

\section{A. Simulation}

We can establish the imaging models of the cylinder by SolidWorks. Simultaneously we can program the formulas of the cylinder's length with MATLAB.

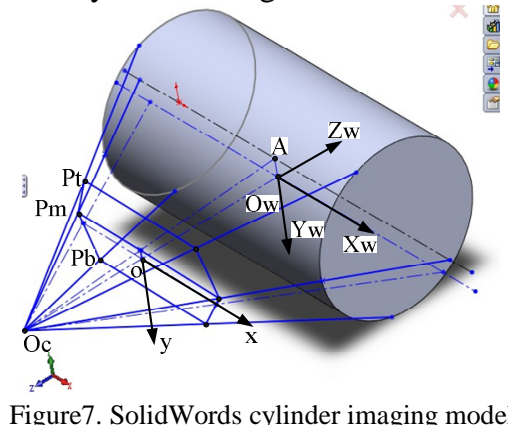

As shown in Fig.7, the imaging model of the cylinder is established by SolidWorks. The image of the upper endpoint, middle endpoint and the lower endpoint in the imaging plane are $p_{t}, p_{m}$ and $p_{b} . O_{c} o$ is equal to $f, O_{c} O_{w}$ is equal to $s$ and $A O_{w}$ is equal to $s_{y}$. The angle between $o x$ and $O_{w} X_{w}$ around $O_{w} Y_{w}$ is $\beta$. The angle between $o x$ and $O_{w} X_{w}$ around $O_{w} Z_{w}$ is $\gamma$. The angle $\gamma$ does not affect the imaging shape but it affects the imaging position. In SolidWorks, we can change the model parameters by dragging the nodes and obtain the coordinates of the angular points of the cylinder image in $\{I\}$. Then we can obtain multiple sets of data about the three-dimension 
objects corresponding to the two-dimension points. These data are reliable.

The flow chart of program with MATLAB is shown in Fig.8.

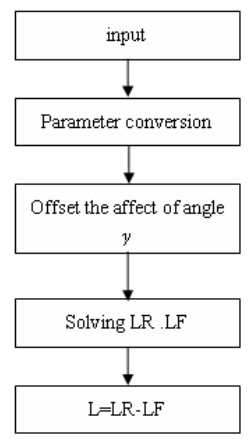

Figure 8. The flow chart of program with MATLAB

(1)Input parameters: The radius of the cylinder $\mathrm{R}$, principal distance $f$ and $s$, deflection angle $\beta$ and $\gamma$, offset $s_{y}$, the coordinates of the four corners of the object imaging $\{I\}$.

(2)Parameter conversion: If the coordinates of the corners are the values in $\left\{I^{\prime}\right\}$, we should convert them from $\left\{I^{\prime}\right\}$ to $\{I\}$.

(3)Offset the affect of angle $\gamma$ : After offsetting the effect of angle $\gamma, x^{\prime}$ is equal to $x \cdot \cos \gamma+y \cdot \sin \gamma$ and $y^{\prime}$ is equal to $-x \cdot \sin \gamma+y \cdot \cos \gamma$.

(4) Solving L: Edit the formulas into program. Then the length of cylinder is calculated.

TABLE I. THE VISION MEASURING RESULTS

\begin{tabular}{cccc}
\hline $\begin{array}{c}\text { Known } \\
\text { conditions }\end{array}$ & 1 & 2 & 3 \\
\hline$f(\mathrm{~mm})$ & 59.83399122 & 85.84446114 & 57.54017706 \\
$s(\mathrm{~mm})$ & 169.92612332 & 195.27282012 & 134.30961675 \\
$R(\mathrm{~mm})$ & 30.00 & 25.22 & 22.86 \\
$s_{y}(\mathrm{~mm})$ & -13.76982952 & 7.86606239 & -8.21001759 \\
$\beta\left({ }^{\circ}\right)$ & 4.81153627 & -6.21654714 & 2.56603759 \\
$\gamma\left({ }^{\circ}\right)$ & 0 & 6.73967415 & 0 \\
\hline$L(\mathrm{~mm})$ & 97.70 & 80.33 & 70.63 \\
\hline
\end{tabular}

TABLE II. THE VISION MEASURING RESULTS

\begin{tabular}{cccc}
\hline Verified result & 1 & 2 & 3 \\
\hline$L_{1}(\mathrm{~mm})$ & 97.7000 & 80.3300 & 70.6300 \\
$L_{2}(\mathrm{~mm})$ & 97.7000 & 80.3300 & 70.6300 \\
$L_{3}(\mathrm{~mm})$ & 97.7000 & 80.3300 & 70.6300 \\
\hline
\end{tabular}

The three sets of data in Table I are obtained by CAD software. The six results of MATLAB program are in Table II. The three results, $L_{1} \sim L_{3}$, are calculated based on the coordinates of the upper endpoint, the middle endpoint and the lower endpoint in image. $L$ is the mean value of the three results. The length values of the cylinder in Table I perfectly match the values in Table II. It demonstrates the correctness of the algorithm established above.

\section{B. Test}

As shown in Fig.8, a fine turning cylinder is used for verifying the algorithms. The cylinder is measured with a vernier caliper. Its length is $38.86 \mathrm{~mm}$ and the diameter is $29.96 \mathrm{~mm}$. We shoot the measured object with the D5000 digital camera. The pictures are processed by the image processing software. The lengths obtained by the software are shown in Table III.

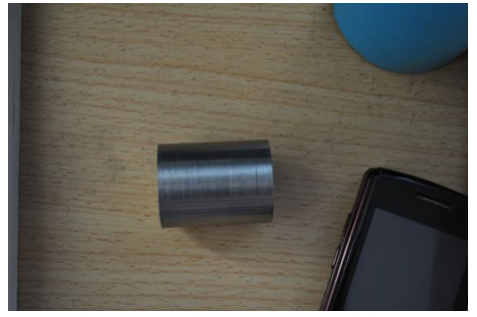

Figure 8. The measured object

TABLE III. THE VISION MEASURING RESULTS

\begin{tabular}{lllll}
\hline Result & \multicolumn{1}{c}{1} & \multicolumn{1}{c}{2} & \multicolumn{1}{c}{3} & \multicolumn{1}{c}{4} \\
\hline \multicolumn{1}{c}{$L(\mathrm{~mm})$} & 38.8465 & 38.8685 & 38.8477 & 38.8553 \\
Absolute error $(\mathrm{mm})$ & -0.0135 & 0.0085 & -0.0123 & -0.0047 \\
Absolute error $(\%)$ & 0.034740 & 0.021873 & 0.031652 & 0.01209 \\
\hline
\end{tabular}

The experiments show that the software can realize the online measuring. The errors are very small and the relative errors are less than $0.1 \%$.

\section{CONCLUSION}

Based on the pinhole camera imaging model, the mathematical modeling method is used to establish the algorithm of three-dimension reconstruction of cylindrical workpiece. The algorithms are verified by simulation and physical test. The image processing software can realize the vision measuring of the cylindrical workpiece in real time. The measuring results are accurate enough to satisfy the practical application.

\section{ACKNOWLEDGMENT}

Supported by Natural Science Fund, China (51275439) and Natural Science Fund of Hebei Province, China (E2012203130).

\section{REFERENCES}

[1] TIAN Weizheng. The research for the three-dimension reconstruction method based on structured light 3D vision theory. Master's degree thesis of computer graphics and digital media disciplines in Jilin University, pp. 3-4,2010

[2] TONG Shuai, XU Xiaogang, YI Chengtao, etc. "Techniques of three-dimension reconstruction based on the visual".Application research of computers, (7), pp.411-2413,2011

[3] HORN B. Shape from shading: a method for obtaining the shape of a smooth opaque object from one view]. United Kingdom: Cambridge, pp. 20-30,1970.

[4] BELHUMEUR P, KRIEGMAN D, YUILLE A. "The bas-relief ambiguity". International Journal of Computer Vision, 35 (1), pp.33-44,1999.

[5] SHI Bo-xin, MATSUSHITA Y, WEI Yi-chen, et al. "Selfcalibrating photometric stereo". IEEE Conference on Computer Vision and Pattern Recognition, pp.1118-1125,2010.

[6] CLERC M, MALLAT S. "Texture gradient equation for recovering shape from texture". IEEE Transactions on Pattern Analysis and Machine Intelligence, 24(4), pp.536-549, 2002.

[7] HARO G, PARDAS M. "Shape from incomplete silhouettes based on the reprojection error". Image and Vision Computing, 28(9), pp.1354-1368, 2010.

[8] HASIN OFF S W, KUTULAKOS K N. "Control stereo". Journal of Mathematical Image and Vision, 81(1), pp.82-104, 2009.

[9] MIKOLAJCZYK K, SCHMID C. "A performance evaluation of local descriptors". IEEE Transactions on Pattern Analysis and Machine Intelligence, 27(10), pp.31-47,2005.

[10] HONG Sisi. The research for Three-dimension modeling technology with light pen. Master's degree thesis of vehicle application engineering in Zhongnan University, pp.49-59, 2011. 